\title{
Integración de procesos, gestión del riesgo y automatización en la gestión de las unidades militares
}

| Fecha de recibido: 7 de julio del 2021 | Fecha de aprobación: 27 de septiembre del 2021 |

Gonzalo

Benítez Lloré

Ingeniero comercial

Fuerza Aérea Ecuatoriana

Ecuador

Rol del investigador: teórico,

experimental y escritura https://orcid.org/0000-0002-2231-9819

$\bowtie$ gbenitez@fae.mil.ec 


\section{Integración de procesos, gestión del riesgo y automatización en la gestión de las unidades militares}

Resumen: La gestión por procesos es implementada en las organizaciones para ordenar actividades secuenciales que generan resultados de valor para los usuarios, aplicarlos en entornos complejos como la gestión de las unidades militares supone un reto administrativo que ha inspirado soluciones manuales e informáticas básicas que no son completamente compatibles con la demanda de rendimiento, integración y precisión en el flujo de información, elementos requeridos en el entorno tecnológico actual para facilitar la toma de decisiones. Las teorías y modelos aplicados a la gestión de riesgos y seguridad operacional en los ámbitos de la aviación, la industria, la medicina, han logrado reducir y mantener niveles de riesgo aceptables para la operación, por lo que pueden ser adaptados y aplicados a los procesos administrativos y de seguridad de las unidades militares para obtener los mismos resultados. En este artículo se plantea un modelo para la implementación de aplicaciones informáticas que fortalezcan los procesos administrativos y de seguridad de las unidades militares de Ecuador, fundamentadas en la combinación de las teorías de gestión por procesos, gestión de riesgos y bases de datos integradas. Al someter esta propuesta a una simulación de procesos se registró un significativo aumento en su eficiencia, que permitió identificar y eliminar los "cuellos de botella", reduciendo el tiempo de ejecución promedio en $94,8 \%$ y la cantidad de personal necesario de siete a dos, a través de la automatización de los procedimientos manuales y de su integración con diferentes departamentos, fortaleciendo la seguridad al acoplar e incluir, de forma visual, las barreras de prevención adoptadas del modelo del queso suizo, para alertar sobre inobservancias a las normas institucionales y los efectos que podrían comprometer la seguridad.

Palabras clave: procesos; automatización; eficiencia; gestión de riesgos.

\section{Process Integration, Risk Management and Automation in Military Units Management}

Abstract: Process management is implemented by organizations in order to classify sequential activities that generate valuable results for users. Applying process management in complex environments such as military units management is an administrative challenge that has inspired manual and basic computer solutions not fully compatible with the demand in terms of performance, integration and precision in the flow of information, elements that are required in today's technological environment to facilitate decision-making. Theories and models applied to risk management and operational safety in the fields of aviation, industry, and medicine have managed to maintain acceptable operational risk levels. Therefore, these models can be adapted and applied to the administrative and security processes involved in military units management to obtain the same results. This article proposes a model for the implementation of computer applications that strengthen the administrative and security processes of Ecuadorian military units, based on the combination of the theories of process management, risk management, and integrated databases. After submitting this proposal to a process simulation, we observed a significant increase in its efficiency, allowing to identify and eliminate "bottlenecks" through the automation of manual procedures and their integration with different departments, reducing the average execution time by $94.8 \%$ and the required staff from 7 to 2 . The proposal also allowed strengthening security by coupling and visually including the prevention barriers adopted from the Swiss cheese model, in order to alert the non-observance of institutional regulations and the derived effects that could compromise security.

Keywords: processes; automation; efficiency; risk management.

Resumo: A gestão de processos é implementada nas organizações para ordenar atividades sequenciais que geram resultados valiosos para os usuários, aplicando-as em ambientes complexos como a gestão de unidades militares, é um desafio administrativo que tem inspirado soluções manuais básicas e informáticas, que não são totalmente compatíveis com a demanda por performance, integração e precisão no fluxo de informações, elementos necessários no ambiente tecnológico atual para facilitar a tomada de decisões. Teorias e modelos aplicados à gestão de riscos e segurança operacional nas áreas de aviação, indústria, medicina, etc. Eles conseguiram reduzir e manter níveis de risco aceitáveis para a operação, de forma que possam ser adaptados e aplicados aos processos administrativos e de segurança das unidades militares para obter os mesmos resultados. Este artigo propõe um modelo de implementação de aplicações informáticas que fortaleçam os processos administrativos e de segurança de unidades militares de Equador, a partir da combinação das teorias de gestão de processos, gestão de riscos e bases de dados integradas. Ao submeter esta proposta a simulação de processos, registou-se um aumento significativo da sua eficiência que permitiu identificar e eliminar "gargalos", reduzindo o tempo médio de execução em 94,8\% e o número de pessoal necessário de 7 para 2, para através do automatização de procedimentos manuais e sua integração com diferentes departamentos, reforçando a segurança por acoplamento e incluindo visualmente as barreiras de prevenção adotadas a partir do modelo do queijo suíço, para alertar sobre o não cumprimento das normas institucionais e os efeitos que podem comprometer a segurança.

Palavras-chave: processos; automação; eficiência; gerenciamento de riscos. 
El rendimiento operativo de las organizaciones es directamente proporcional a su capacidad de gestionar la información, tanto interna como externa, así como sus procesos (Prajogo et al., 2018), haciendo imprescindible la introducción y constante actualización de tecnologías que contribuyan a la administración eficiente, integrada y coordinada para elevar el nivel de competitividad en sus productos o servicios.

En este contexto, al hablar de la gestión de las instituciones militares, se evidencia un elevado nivel de complejidad, puesto que requiere cumplir y hacer cumplir procedimientos operativos y administrativos, enmarcados en las normativas legales que rigen su funcionamiento y que pueden generar responsabilidades ante su inobservancia, por ejemplo, la administración de personal que está en constante rotación y la custodia de recursos críticos como el armamento y los medios militares empleados para la seguridad y la defensa del país.

Ante este escenario, continuamente se han implementado procesos orientados a optimizar la gestión de las unidades militares; sin embargo, hay quienes carecen de un nivel de automatización compatible con la tecnología disponible en la actualidad, dando paso a la ocurrencia de errores administrativos y vulnerabilidades en los sistemas de seguridad.

De aquí surge la importancia de considerar la implementación de soluciones automatizadas para la ejecución de tareas que no requieren, necesariamente, de la intervención humana (Figueroa-García et al., 2017), como una aproximación para fortalecer la gestión, aumentar las capacidades de control y prevención, así como para mejorar la comunicación y el trabajo integrado entre los actores de los procesos institucionales. Por ello, es automatización, la opción más viable para combinar estos requerimientos $\mathrm{y}$, al mismo tiempo, mejorar la experiencia de los usuarios.

Esta investigación fue motivada por la identificación de inconvenientes en la gestión de la información en los procesos administrativos y de seguridad de las unidades militares de la Fuerza Aérea Ecuatoriana (FAE), especialmente, en lo que se refiere a la del talento humano, seguridad operacional y física. Estas debilidades tienen la capacidad potencial de afectar el normal desarrollo de los procesos, produciendo errores que podrían, no solo ser observados por organismos de control interno y externo a la institución, sino vulnerar los sistemas de seguridad física e integrada (Evans et al., 2019).

El objetivo de este trabajo académico es plantear un modelo de Sistema Integrado de Gestión para el diseño y aplicación de soluciones informáticas en la gestión de procesos en las unidades militares (Ministerio de Defensa Nacional, 2018a), combinando el rediseño de procesos, la prevención de riesgos y la automatización en un entorno de base de datos integrada, encaminados a elevar la eficiencia en la gestión administrativa y de seguridad; el intercambio de información en tiempo real; el trabajo integrado entre departamentos; la mejora de la experiencia de los usuarios y la integridad de los registros, los indicadores y las estadísticas.

\section{Descripción de la metodología}

Para identificar las debilidades en la gestión de la información de los procesos administrativos y de seguridad en las unidades militares y concebir una propuesta aplicable para su solución, se realizó una revisión teórica sobre el rediseño de procesos y su impacto en la organización al mejorar su desempeño; de los modelos de gestión de riesgos para la seguridad operacional y su aplicación al ámbito administrativo; así como del diseño de bases de datos integradas para alcanzar las capacidades que demanda esta propuesta.

Este modelo combinado se enmarca, principalmente, en las teorías de bases de datos integradas de Ricardo (2009); la gestión por procesos de Pérez (2004); la mejora en el rendimiento de los procesos de Raynus (2011) y su medición, a través de indicadores de desempeño clave (IDC), planteados por Van Der Aalst et al. (2016); el modelo cooperativo conceptual para el diseño de procesos, digitalización e innovación de Sehlin et al. (2019) y el modelo del queso suizo de Reason (2009), y de sus casos de aplicación, expuestos en el artículo "Managing Risk Through Layers of Control" de Lyon y Popov (2020). 
También se han considerado estudios sobre el impacto de la introducción de sistemas para el análisis de datos (Hu et al., 2018) y casos en los que se ha implementado el modelo del queso suizo para la gestión del riesgo, la investigación y la prevención en los campos de la seguridad operacional y ocupacional en la aviación (Mat Ghani \& Zheng Yi, 2018), la medicina (Noh et al., 2020) y la industria (Shokouhi et al., 2019).

A partir de esta información, se desarrolló e implementó un software piloto con algunas de las funcionalidades básicas que se formulan en este artículo, integrando los procesos y el intercambio de información automática entre el Departamento de talento humano y los departamentos Seguridad integrada, el Centro de salud y el Sistema de seguridad física de la unidad militar, facilitando el seguimiento de los procesos en tiempo real y la generación de reportes y alertas automáticas ante inconsistencias en los registros 0 incumplimientos a las normas vigentes, es decir, que se adaptó el modelo de queso suizo a una interfaz amigable para la identificación oportuna de fallas y la gestión del riesgo en los procesos administrativos y de seguridad.

Se instruyó al personal del Departamento de talento humano del ala de combate n. ${ }^{\circ} 21$ de la FAE para que lo administre e ingrese todas las solicitudes referentes al subproceso Monitoreo de personal, en forma paralela a los procedimientos que se ejecutan manualmente. Es importante recalcar que, a partir del ingreso de esta información, se actualizan y se modifican los registros de los otros departamentos de forma inmediata, por ejemplo, si una persona era transferida hacia otra unidad militar, los administradores debían desactivar su cuenta, esto provocaba la actualización automática de los reportes y los registros del personal disponible en los otros departamentos, así como la anulación de autorizaciones de acceso y habilitaciones.

Posteriormente, se realizó la descarga y análisis de los datos obtenidos en el periodo de un año, para establecer de forma cuantificable sus IDC, que fueron ingresados en el programa de simulación de procesos libre "BPSimulator" (Prolis lab2k, 2019) el cual fue configurado de acuerdo a las observaciones realizadas a través de una investigación empírica para identificar las principales causas de los inconvenientes presentados en su desarrollo, obteniendo así información y un diagnóstico sobre el impacto en el rendimiento de la totalidad del subproceso bajo criterios, condiciones y demoras aleatorias similares a las observadas empíricamente, como una aproximación al entorno real para estimar el impacto en términos de optimización, con miras al rediseño de los procesos que serán automatizados (Heinrich et al., 2017).

\section{Rediseño de la gestión por procesos}

Se considera que los procesos inefectivos e ineficientes existen porque no son diseñados como un todo, sino que son ajustados de forma improvisada de acuerdo a la dinámica del trabajo y a los casos especiales que se presentan en las organizaciones. Generalmente este fenómeno se proyecta en el tiempo, trascendiendo de generación en generación (Laguna \& Marklund, 2018).

Un proceso está definido como la "secuencia [ordenada] de actividades [repetitivas] cuyo producto tiene valor para su usuario o cliente" (Pérez, 2004). El autor también hace referencia al concepto de un sistema como un "conjunto de elementos interrelacionados que persiguen un objetivo común" (Pérez, 2004) y más adelante en su obra aborda el modelo de SIG, aplicado en las organizaciones, combinando la gestión de la calidad, el medio ambiente, los riesgos laborales, entre otros, como factores estratégicos adoptados por las empresas para elevar su eficiencia y competitividad.

Esta combinación multidimensional puede ser adaptada a las necesidades de las unidades militares para obtener un impacto similar. En el caso particular de esta propuesta, se ha reorientado a un SIG que combina la calidad de los procesos administrativos y el fortalecimiento de la seguridad. La implementación de esta herramienta permite mejorar la gestión organizacional, enfocándola a la excelencia en el servicio para los usuarios, en este caso, los comandantes, jefes departamentales y el personal militar en general.

Un estudio realizado sobre la metodología de la mejora de los procesos en universidades australianas 
plantea un modelo que ayuda a entender la excelencia en el servicio, la cual se enfoca en cuatro ejes: 1) la estrategia anclada a la excelencia en el servicio, 2) la cultura modificada hacia modalidades "self-service”, 3) los sistemas y 4) los procesos como vehículo para entregar servicios eficientes y la medición del desempeño, tanto para establecer el grado de operacionalización de la excelencia en el servicio, como para utilizarla como evidencia en la toma de decisiones (Ciancio, 2018).

Es importante considerar que las modificaciones que se implementen en los procesos no necesariamente garantizan una mejora en la eficiencia o un impacto favorable para todos sus participantes. Al adoptar soluciones improvisadas o aisladas, se corre el riesgo de comprometer los IDC de otro proceso o la carga de trabajo de otro departamento o usuario, haciéndolo inefectivo o ineficiente.

El rediseño de procesos requiere de un enfoque integral y un análisis permanente, ya sea observado, o estimado, de los indicadores de desempeño aplicados a la solución propuesta para asegurar que realmente se los está optimizando en su conjunto. En el caso de esta investigación, el rediseño de procesos ha ido acompañado de IDC en parámetros de tiempo, costo y calidad (Van Der Aalst et al., 2016).

Otro aspecto a considerar es la necesidad de sustituir el paradigma tradicional orientado a la estructura organizacional, donde los procesos se ejecutan individualmente en cada departamento, para reemplazarlo por el nuevo paradigma orientado al proceso, en el que se lo ejecuta de forma transversal en la organización, con la participación de varios departamentos, conceptualizándose como procesos multifuncionales (Raynus, 2011).

Para efectos de esta investigación se seleccionó como muestra al subproceso "Monitoreo de personal" (Dirección de planificación y gestión estratégica de la Fuerza Aérea Ecuatoriana, 2018), constituyéndose como uno de los más demandantes para los departamentos de Talento Humano de las unidades militares de la FAE, debido a que se ejecutan procedimientos complejos y se generan cantidades considerables de documentos, siendo catalogados de la siguiente manera:
- Registro de personal.

- Partes de personal.

- Roles de servicios.

- Régimen de personal.

- Sistema de gestión documental.

Todos ellos generan estadísticas, informes y proyecciones que contribuyen al control y cumplimiento de normas legales e institucionales aplicadas a la gestión del talento humano.

A través de la investigación empírica se identificaron afectaciones a los IDC de este subproceso y se pudo determinar que su causa predominante fue la excesiva intervención manual en tareas que pueden ser ejecutadas por herramientas informáticas. En lo que respecta a los tiempos de ejecución se registraron demoras variables en cada etapa del subproceso (cuellos de botella) provocados por la intervención de personas con excesiva carga de trabajo que colaboran en otras tareas del departamento. A esta demora se suma el tiempo transcurrido durante el transporte de la documentación entre varios departamentos o funciones durante el desarrollo del subproceso.

Para el cálculo de los costos se consideró el generado por la impresión de los documentos requeridos en el subproceso. Se ha excluido el gasto económico por remuneración debido a la naturaleza y características de la institución militar, sin embargo, si se considera una tasa promedio de tiempo empleado por persona para cada tarea.

El indicador de calidad del subproceso marca la cantidad de inobservancias en los plazos de estos trámites administrativos, considerado en este caso como un error debido a que la exigencia institucional es que la legalización de estos documentos sea cumplida de forma anticipada, es decir, máximo hasta la fecha de inicio que estipula la solicitud presentada.

Una vez establecidos estos IDC, se recopilaron los datos del software piloto, correspondientes a un periodo aleatorio de un año, entre el 15 de abril del 2019 y 15 de abril del 2020. Los resultados mostraron un total de 4.141 solicitudes registradas, las cuales fueron presentadas en 310 días, que equivalen a un promedio de 13,4 solicitudes presentadas a diario; sin embargo, se 
legalizaron 4.121 en 236 días, lo que representa un promedio de 17,5 legalizaciones diarias.

La diferencia entre estos promedios pone de manifiesto que el esfuerzo realizado por el personal involucrado en el subproceso no es suficiente para cubrir la totalidad de las solicitudes presentadas, debido a que una solicitud se puede presentar en cualquier fecha y hora, mientras que su procesamiento es ejecutado en días y horario laborables, disponiendo de tiempos menores que, a su vez, generan retrasos en su cumplimiento.

De las 4.141 solicitudes presentadas al Departamento de Talento Humano, 791 fueron ingresadas de forma extemporánea, la ocurrencia de estos eventos se distribuyó en 250 días. Lo que significa que el mismo número de reportes diarios e informes de situación de personal que fueron emitidos en el periodo analizado, presentaron alguna inconsistencia, es decir, un $68,5 \%$ al año.

Estos resultados, así como la información obtenida a través de la investigación empírica fueron ingresados al simulador de procesos libre "BPSimulator" con la finalidad de obtener un parámetro de tiempo de ejecución de este que se aproxime al observado. Considerando que se desconoce el tiempo transcurrido desde que el solicitante inicia el subproceso hasta la presentación de su documento en el Departamento de talento humano, se configuró el simulador para que seleccione aleatoriamente demoras en el rango de 15 a 120 min en los procedimientos de esta fase y combinarlos con los registrados durante la legalización, registro manual y archivo.

Se incluyeron puntos de control en la simulación que permitieron medir el tiempo de ejecución del subproceso en varias etapas de la secuencia, así se obtuvieron las referencias de este parámetro en las tareas de legalización del jefe del Departamento de talento humano, actualización del parte diario de personal, actualización del rol de servicios (personal de guardia) y el archivo de los documentos como punto de finalización.

La simulación determinó que el tiempo de ejecución promedio de una solicitud, desde que es iniciada hasta que es archivada al final del subproceso, es de 06:35:41 y emplea siete personas (véase figura 1), lo que representó un tiempo promedio de 0:56:25 por cada una. Considerando que el periodo laborable es de 08:00:00, este resultado pone de manifiesto la razón por la cual se da la acumulación de las tareas de actualización de los registros y estadísticas del personal, las cuales deben ser ejecutadas en horarios extendidos e incluso al día siguiente. Este fenómeno es una de las principales causas de las inconsistencias y demoras en los registros y estadísticas del subproceso.

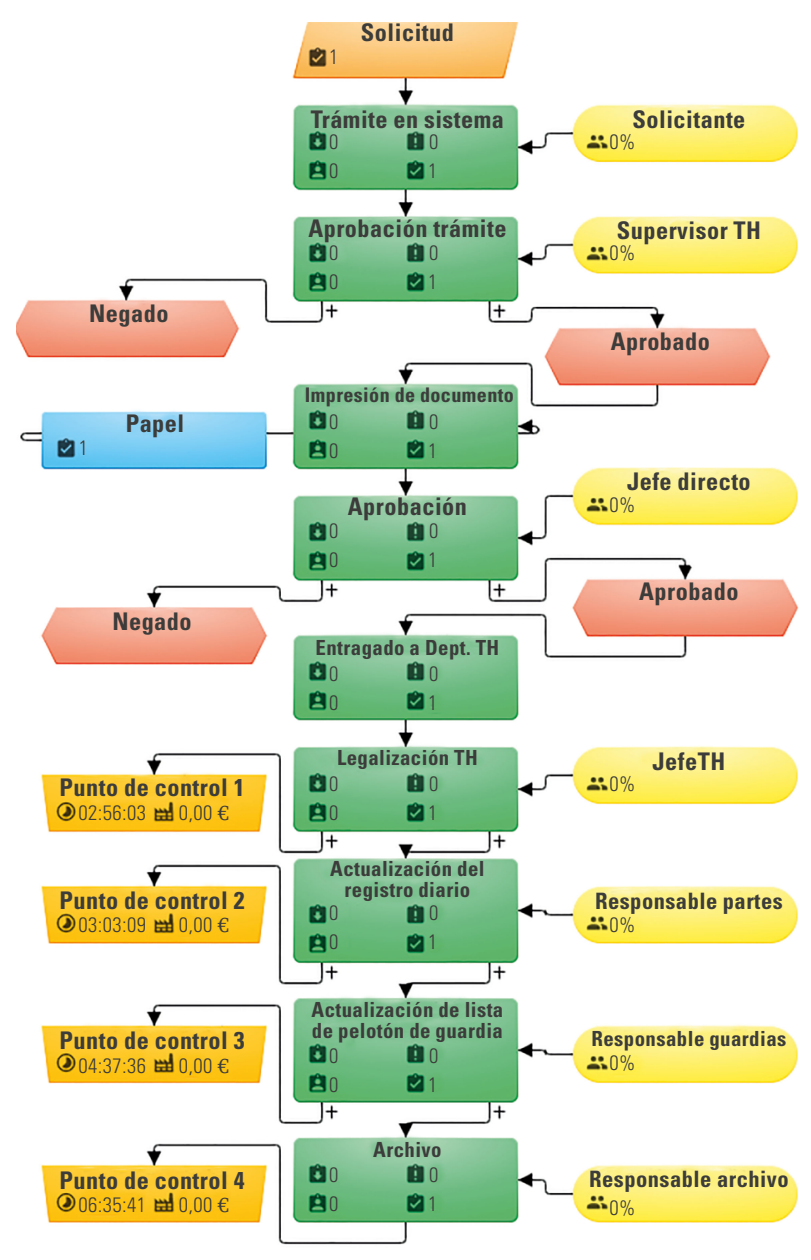

Figura 1. Subproceso de monitoreo de personal ingresado en "BPSimulator" (1 solicitud)

Fuente: BPSimulator.

Al configurar el simulador para el procesamiento de 13 solicitudes diarias, de acuerdo con la información arrojada por el software piloto, se observó el fenómeno de retraso explicado anteriormente y el indicador 
de longitud de cola en el horario laborable (véase figura 2). Los resultados indican que el $25 \%$ del tiempo total del subproceso es de espera y el $40 \%$, de transporte, es decir, la demora de transferir el documento de una función a otra, además que de 13 trámites iniciados solo dos llegaron al fin del subproceso en el mismo día, las tareas pendientes para el día siguiente fueron:

- Seis aprobaciones del supervisor de talento humano.

- Dos legalizaciones del jefe de talento humano.

- Tres de la lista del personal de guardia (rol de servicios).

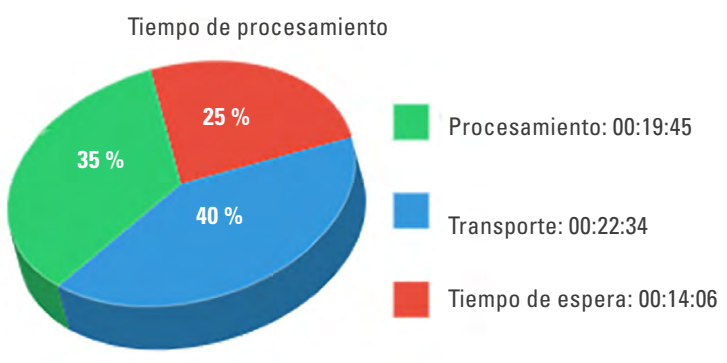

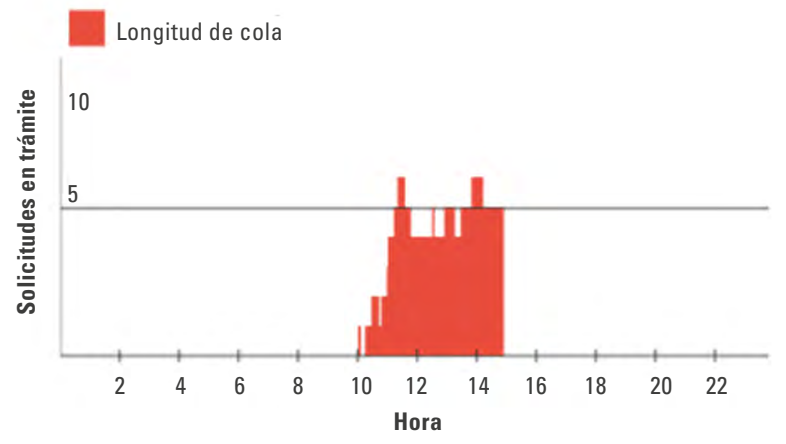

Figura 2. Resultado del subproceso de monitoreo de personal ingresado en "BPSimulator" (13 solicitudes) Fuente: BPSimulator.

Al rediseñar el subproceso, tomado como ejemplo y utilizando el simulador para ajustarlo a la implementación de un software que contribuya a simplificary mejorar sus IDC, se evidenció una significativa mejora en términos de eficiencia. Las principales modificaciones fueron: la eliminación de la intervención de cinco personas, así como de los tiempos de espera y transporte que no contribuyen al procesamiento de los trámites (véase figura 3), dando como resultado la reducción del tiempo empleado por persona a 0:10:10.

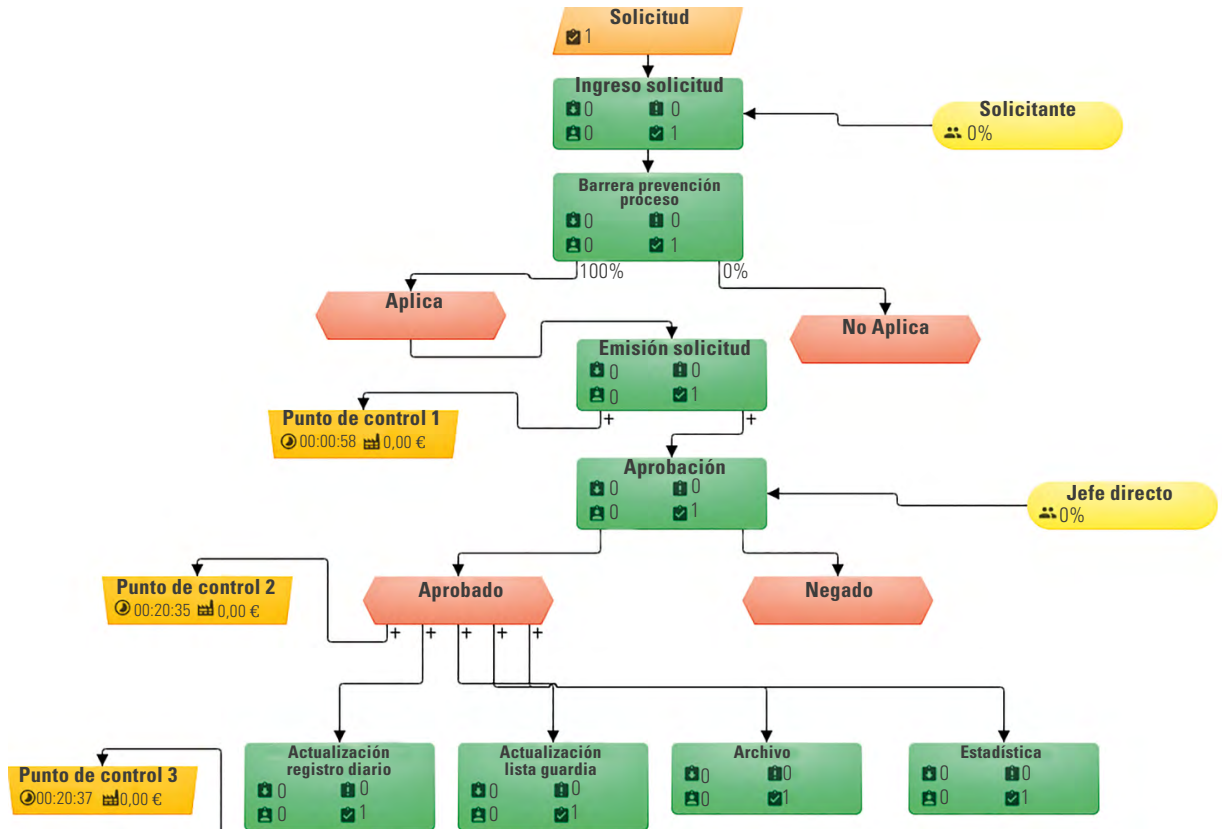

Figura 3. Rediseño propuesto para el subproceso de monitoreo de personal ingresado en "BPSimulator" (1 solicitud) Fuente: BPSimulator. 
Los resultados de la simulación arrojaron una reducción del tiempo de ejecución del subproceso de 06:35:41 a 0:20:37, es decir, de un 94,8 \%, aun cuando fue introducida una validación de solicitudes automática como barrera de prevención ante errores o inobservancias a las normas institucionales. En este caso, el tiempo que puede afectar la normal ejecución del subproceso recae sobre la revisión y aprobación de la solicitud por parte del jefe directo del solicitante. Después de ejecutar esta aprobación todos los registros y estadísticas se actualizan automática y casi instantáneamente como se visualiza en las

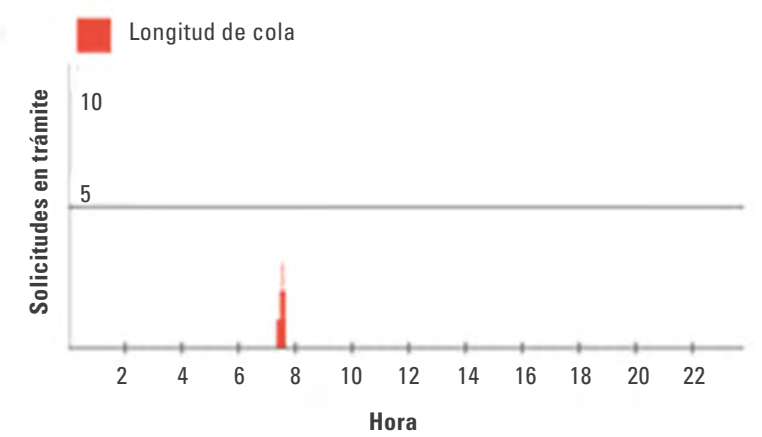

mediciones de tiempo de los puntos de control 2 y 3 , actualizando, los registros de otros departamentos automáticamente.

Los resultados de la simulación para el procesamiento de 13 solicitudes diarias (véase figura 4) mostraron una significativa reducción de la acumulación de los trámites (longitud de cola). El tiempo de espera se redujo al $5 \%$ del tiempo total de ejecución y el de transporte se elevó hasta $87 \%$. Vale la pena recalcar que este tiempo depende de la demora en la aprobación por parte del jefe directo del solicitante. Se observó, también, que los 13 trámites iniciados lograron concluirse el mismo día.

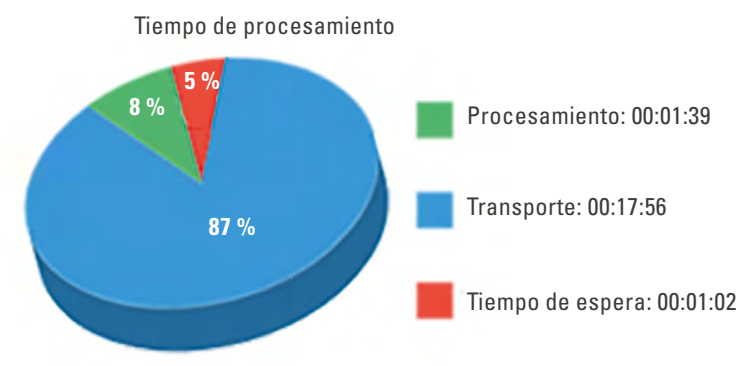

Figura 4. Resultado de la propuesta de rediseño del subproceso de monitoreo de personal ingresado en "BPSimulator" (13 solicitudes) Fuente: BPSimulator.

\section{Automatización de la gestión en un entorno de base de datos integrada}

La correcta implementación de soluciones informáticas para análisis de datos en los procesos de una organización lleva implícito un impacto favorable para la eficiencia en la gestión y control, pues pone a disposición herramientas para generar estadísticas, cálculos y reportes de forma más ágil y exacta que las herramientas de uso común como las tablas de Excel (Hu et al., 2018).

Estas aplicaciones permiten el monitoreo remoto y en tiempo real (Jamil et al., 2020), generando un flujo de información permanentemente actualizado hacia los departamentos responsables de los procesos, lo que les permite trabajar e interactuar de forma colaborativa, modalidad que tiene un impacto favorable en el rendimiento de la organización (Askari et al., 2020).
La necesidad de implementar este tipo de soluciones en las organizaciones de gran tamaño radica en la gestión y procesamiento de grandes cantidades de información, por ejemplo, en las instituciones de salud (Amaechi et al., 2018), caracterizadas por ejecutar procesos críticos y que demandan de exactitud y velocidad para la toma de decisiones. Este mismo requerimiento se aplica a los procesos administrativos y de seguridad (Brooks et al., 2020) de las unidades militares para hacerlos más eficientes.

En este contexto se plantea la aplicación de un modelo de base de datos integrada, el cual cuenta con un único repositorio de datos que puede ser utilizado de manera simultánea por varios departamentos y usuarios, no es exclusivo de un solo participante y se constituye como un recurso compartido de la organización (Ricardo, 2009). Las principales ventajas de este modelo según Ricardo (2009) son: 
1. La compartición de datos entre todos los departamentos de acuerdo a la competencia de sus procesos.

2. El control de redundancia de datos, evitando registros duplicados que puedan generar inconsistencias en las estadísticas.

3. Consistencia de datos, pues al existir un solo dato que respalde las estadísticas, cualquier actualización de este se lo realizará una sola vez y afectará a todos sus usuarios.

4. Estándares de datos mejorados que permiten mantener un solo formato de acuerdo a las necesidades institucionales.

5. Se fortalece la seguridad de los datos mediante el control y restricción de acceso u operaciones no autorizadas en la base de datos.

6. Se fortalece la integridad de datos mediante la posibilidad de crear alertas y restricciones ante comportamientos no deseados para que puedan ser contrarrestados anticipadamente.

7. Se establece un equilibrio de los requisitos de los usuarios, evitando que entren en conflicto con las necesidades de otros usuarios.
8. El tiempo para desarrollar actualizaciones o mejorías al software se reduce, debido a que se cuenta con datos relacionados a la organización para su uso inmediato.

9. Mejora la accesibilidad a los datos a través de interfaces interactivas y amigables.

10. Al centralizar la base de datos, los costos de operación y mantenimiento se reducen a una sola instalación física.

11. Mejores procedimientos de respaldo y recuperación, pues al mantener una única base de datos se pueden programar y ejecuta operaciones de respaldo automático de un solo servidor.

Las ventajas que ofrece este modelo pueden ser explotadas en diferentes aspectos de acuerdo a las necesidades de cada departamento (véase figura 5), elimina procedimientos manuales y sincroniza las estadísticas y reportes, pues si un departamento ingresa o actualiza un dato, este cambio se verá reflejado en los indicadores y reportes de los otros departamentos participantes de forma automática.

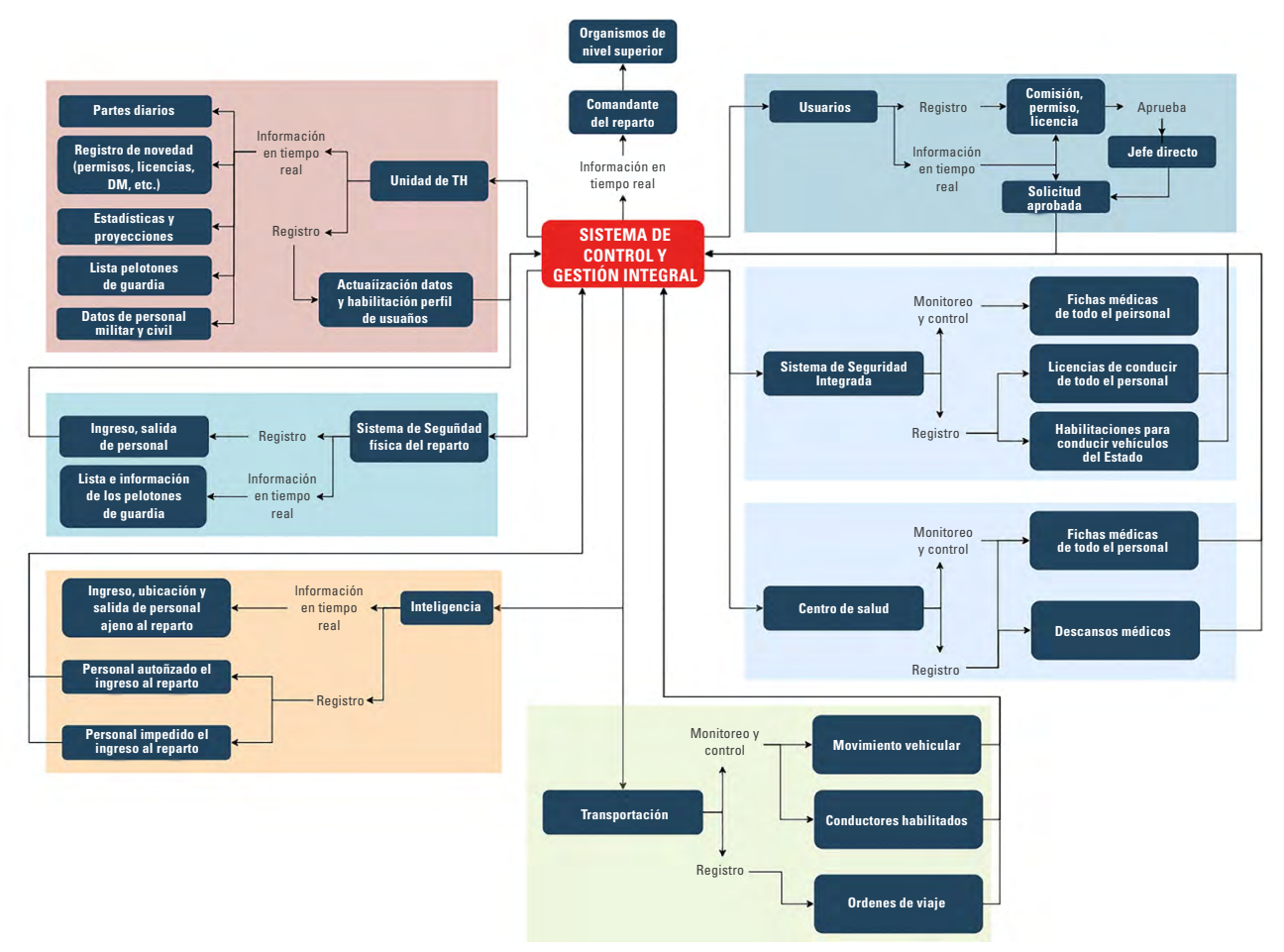

Figura 5. Capacidades de la base de datos integrada y su relación con los departamentos de la organización. Fuente: elaboración propia. 
La implementación del software OVFAE en el 2015, concebido a partir de la "Propuesta de diseño e implementación de un Sistema de información gerencial aplicado a la operaciones aéreas y evaluación de pilotos para el Escuadrón de Combate n. ${ }^{\circ} 2.313$ de la Fuerza Aérea Ecuatoriana" (Benítez, 2013), constituye un ejemplo de las potenciales prestaciones que ofrece la adopción de un modelo similar y que puede ser aplicado a los procesos administrativos y de seguridad.

Este diseño elimina la duplicidad, inconsistencias y errores en los registros, además permite un seguimiento en tiempo real de los indicadores en todos los niveles de la organización, la creación de alertas que pueden ser disparadas ante la detección de un comportamiento anormal y su notificación vía correo electrónico para la adopción de su acción correctiva de forma anticipada.

Es importante recalcar que, al introducir soluciones tecnológicas a la gestión por procesos, se fortalece la estandarización de las tareas y sus indicadores, que no se verían afectados en su integridad o rendimiento, considerando el entorno variable característico de las unidades militares debido a su alta rotación de personal (Nikolaidou et al., 2001).

\section{Adaptación del modelo del queso suizo en entornos informáticos}

En el ámbito de la seguridad es conocido que la probabilidad de cometer errores se encuentra latente por naturaleza, en todo entorno o actividad que involucre la intervención del ser humano. Importantes autores como James Reason (1990) han desarrollado y publicado, tanto estudios, como modelos para explicar este fenómeno, prevenirlo y contrarrestar sus efectos, entre los cuales se destaca el "modelo del queso suizo". Su amplio espectro de aplicación ha generado resultados positivos para la seguridad operacional y ocupacional en la aviación (Mat Ghani \& Zheng Yi, 2018), la medicina (Noh et al., 2020), la industria (Shokouhi et al., 2019), etc.
El modelo del queso suizo es considerado una herramienta poderosa en el pensamiento de las barreras preventivas o líneas de defensa (Reniers et al., 2020), las cuales se representan como planos que contienen ventanas de oportunidad creadas como consecuencia de actos inseguros y brechas en los sistemas de defensa. Estas áreas de permeabilidad varían constantemente en su ubicación y tamaño por la gran cantidad de variables impredecibles que afectan a su respectivo plano. Bajo ciertas circunstancias estas brechas pueden alinearse para formar una trayectoria directa hacia la ocurrencia de un accidente (Reason, 1990).

La aplicación de este modelo a casos de estudio o investigación de accidentes (Lyon \& Popov, 2020) contribuye al análisis, comprensión e identificación de las fallas en las capas de protección que causaron el accidente (Akuh \& Atombo, 2019), siendo un aporte valioso y puntual para la seguridad operacional y permitiendo fortalecer las medidas preventivas para interrumpir la cadena de eventos que conducen a un desastre (Larouzee \& Le Coze, 2020).

Los procesos administrativos, que por lo general no acarrean un riesgo catastrófico, sí tienen la capacidad de generar responsabilidades legales y comprometer la seguridad en los ámbitos operacional y físico de las unidades militares, más aún en el escenario actual donde han proliferado amenazas asimétricas (Ministerio de Defensa Nacional, 2018b), cuyos efectos ya han afectado a las instituciones militares. Entonces, ¿por qué no dar el mismo tratamiento de prevención a estos procesos?

En la figura 6 se aprecia la adaptación del modelo del queso suizo de Reason al subproceso de verificación de documentos exigidos por las normas internas y externas a la institución, previo a la salida de vehículos institucionales. En este esquema, los controles existentes se han establecido como barreras preventivas que dependen de dos organismos: el Departamento de seguridad integrada y el Centro de salud. La falla en cualquiera de estas barreras representa la inobservancia de alguna norma, por lo que son alertadas en color rojo y deben ser corregidas inmediatamente después de ser detectadas. 
Aun cuando el subproceso de verificación es claro y sencillo, su aplicación presenta vulnerabilidades que favorecen el cometimiento de errores, pues la persona que lo ejecuta solo tiene acceso al documento del chequeo médico diario que presenta el conductor (Chequeo pre-manejo), es decir, una de las cuatro barreras de prevención existentes para garantizar que un conductor se encuentre habilitado.

Esta situación se presenta debido a que, en el punto de control, no se dispone de acceso a la información de todos los organismos que participan en la habilitación del conductor. El hecho de que la tarea sea manual (presentación de un documento) y de que esto ocurra en un entorno donde existe una altísima rotación de personal (turnos de 3 horas), constituyen condiciones propicias para la toma de decisiones subjetivas y no estandarizadas.

Aumentar los documentos a presentar por el conductor y cumplir con sus respectivas verificaciones para disminuir la permeabilidad en las cuatro barreras de prevención establecidas institucionalmente, acrecentaría el tiempo de ejecución de este subproceso y, consecuentemente, la carga de trabajo, tanto para el conductor, como para la persona que hace la verificación. Es aquí donde se evidencia la necesidad de una aplicación informática para acelerar el desarrollo del subproceso y facilitar la toma de decisiones de forma inmediata y estandarizada.

\section{Adaptación del modelo del queso suizo a las verificaciones efectuadas para conducir un vehículo institucional}

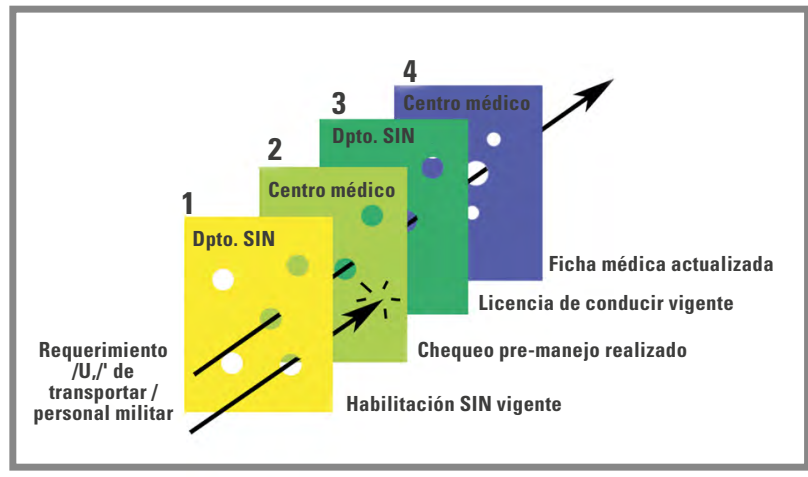

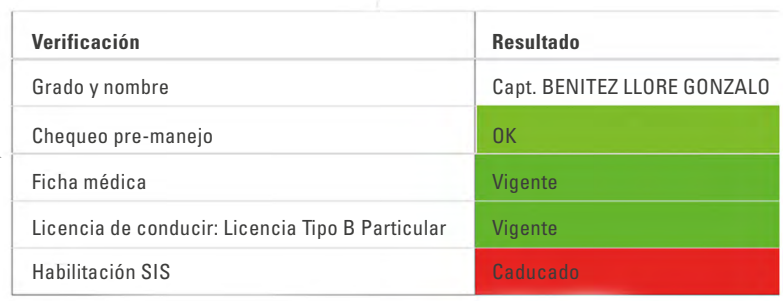

Aplicación del modelo del queso suizo a un software de control administrativo y de seguridad

Figura 6. Aplicación del modelo del queso suizo al diseño de un sistema informático Fuente: elaboración propia.

Adaptar este modelo a través de una aplicación informática permite vincular los datos de los organismos participantes en el subproceso, información que estaría disponible tan solo con ingresar el número de identificación del conductor que se presente en el punto de control de la unidad militar. Así se puede alertar inmediatamente sobre alguna inhabilitación en cualquiera de las cuatro barreras de prevención.

En este caso, tomado como ejemplo la decisión estandarizada que sugiere el sistema a cualquier usuario, mediante una indicación de incumplimiento, se busca evitar que el conductor haga uso de un vehículo institucional por estar inhabilitado, puesto que, ante la ocurrencia de un incidente o accidente, pondría al descubierto inobservancias administrativas con el potencial de generar responsabilidades legales u obstáculos en la aplicación de seguros.

Este ejemplo demuestra la importancia de compartir información entre los organismos que participan en el mismo proceso, lo que fortalece la gestión administrativa y la seguridad. Esta carga de información debe observar un diseño amigable, resumido, en 
tiempo real y adaptado a un modelo de prevención de riesgos, facilitando la toma de decisiones estandarizadas y alineadas a las normas institucionales.

Este modelo puede ser aplicado a otros procesos de seguridad de las unidades militares, como el control de acceso de personal a las instalaciones (Song et al., 2018), en el que se conectaría a los departamentos responsables de la seguridad y recursos humanos para la emisión de autorizaciones de ingreso al personal, de acuerdo con perfiles que permitan adoptar protocolos de seguridad, teniendo en cuenta el nivel de riesgo que representan, así como el monitoreo durante su permanencia, el registro de salida y un historial de visitas.

Los resultados expuestos evidencian los beneficios en la gestión y seguridad que se pueden alcanzar en cualquier proceso de las unidades militares, aplicando un modelo de SIG que combina la calidad de los procesos administrativos y el fortalecimiento de la seguridad mediante el rediseño de procesos, la gestión de riesgos y la automatización en un entorno de base de datos integrada.

\section{Resultados}

La simulación del rediseño del subproceso "Monitoreo de personal" utilizado en este ejemplo permite hacer una comparación cuantitativa de los IDC en parámetros de tiempo, costo y calidad (véase tabla 1), para medir la mejora en términos de eficiencia, como resultado de la implementación del modelo propuesto en esta investigación.

Tabla 1

Mejoras en términos de eficiencia en el subproceso de monitoreo de personal

\begin{tabular}{|c|c|c|}
\hline Parámetro & Proc. Manual & Proc. Automatizado \\
\hline Registros con inconsistencias en un año & $68,5 \%$ & $1,7-2,2 \%$ \\
\hline Costo anual impresiones (aprox.) & 85,82 USD & 0,00 USD \\
\hline Tiempo de ejecución del subproceso & $06: 35: 4$ & $0: 20: 37$ \\
\hline Cantidad de personas empleadas en el subproceso & 7 & 2 \\
\hline Promedio de tiempo empleado por persona en el subproceso & $0: 56: 25$ & $0: 10: 10$ \\
\hline
\end{tabular}

Fuente: elaboración propia.

En cuanto al subproceso de verificación de documentos previo a la salida de vehículos institucionales, no se contó con registros que puedan arrojar una medición cuantitativa de IDC; sin embargo, se exponen las capacidades que brinda esta herramienta en el punto de control de la unidad militar desde un enfoque cualitativo.

Tabla 2.

Mejoras cualitativas en el subproceso de verificación de documentos previo a la salida de vehículos institucionales

\begin{tabular}{l|c|c}
\multicolumn{1}{c|}{ Parámetro } & Proc. Manual \\
\hline $\begin{array}{l}\text { Cantidad de barreras de prevención visualizadas por el usuario Integración } \\
\text { de información entre departamentos participantes (enfoque multifunción) }\end{array}$ & 1 & Proc. Automatizado \\
\hline $\begin{array}{l}\text { Integración de información entre departamentos participantes } \\
\text { (enfoque multifunción) }\end{array}$ & No \\
\hline Información disponible en tiempo real & No \\
\hline
\end{tabular}




\section{Discusión}

El rediseño de la gestión por procesos permite implementar SIG para la administración eficiente, segura y ajustada a las necesidades de las unidades militares, reorientándolos a un enfoque multifunción de ejecución transversal en la organización que fortalezca la comunicación y el trabajo integrado entre sus actores. En este contexto, es importante considerar la evaluación permanente de los IDC dentro de los procesos modificados para evaluar y evidenciar de forma cuantitativa el impacto en la eficiencia ante su implementación.

La automatización de la gestión por procesos a través de un entorno de base de datos integrada permite la ejecución y monitoreo de procesos multifuncionales en las unidades militares, lo que implica una mejora sustancial en la comunicación e intercambio de información entre sus departamentos; un aumento significativo de la eficiencia en cuanto al procesamiento de datos, tiempos de ejecución y uso de recursos; además de la estandarización de las tareas y sus indicadores frente a un entorno de alta rotación de personal.

La adaptación del modelo del queso suizo a los procesos administrativos y de seguridad es un aporte valioso que permite identificar y analizar las fallas en las capas de protección, facilitando la adopción de medidas preventivas para interrumpir la cadena de eventos que conducen a situaciones no deseadas, en el caso de esta investigación, a la inobservancia de las normas legales y vulnerabilidades en la seguridad, de la misma forma que ha sido aplicado en los campos de seguridad operacional y ocupacional en la aviación (Mat Ghani \& Zheng Yi, 2018), la medicina (Noh et al., 2020), la industria (Shokouhi et al., 2019), etc.

La combinación de estos tres elementos como un SIG, el cual fue ingresado en el simulador de procesos "BPSimulator" para su evaluación, demostró un impacto positivo en cuanto a su eficiencia en el subproceso "Monitoreo de personal" tomado como muestra, debido a una reducción en su tiempo de ejecución promedio en un $94,8 \%$, sus costos de impresión de 85,82 USD a 0 USD, la tasa de horas empleadas por hombre de 0,94 a 0,17 y los registros diarios de personal con inconsistencias de 68,5 \% a 1,7-2,2 \% al año. Estos resultados evidencian la optimización del proceso de forma integral, aún con la introducción de barreras de prevención.

En cuanto al subproceso de verificación de documentos previo a la salida de vehículos institucionales, la implementación de este modelo posibilita la visualización, mediante una interfaz amigable para los usuarios, de todas las barreras de prevención adoptadas institucionalmente y su estado, en cuanto a su permeabilidad en tiempo real, permitiendo establecer alertas y notificaciones mediante correos electrónicos, lo que contribuye a la toma de decisiones estandarizadas y alineadas con la normativa legal.

Los resultados alcanzados mediante el uso del software piloto puesto en funcionamiento coinciden con la evaluación cuantitativa de los resultados del estudio sobre la implementación de un sistema de iniciativa mixta que respalda los datos integrados en los flujos de trabajo, donde se pone de manifiesto que el uso de software dedicado a facilitar la exploración de los datos ofrece resultados significativamente mayores en cuanto a la visualización y la rapidez para completar estas tareas, en comparación con el uso tradicional de programas como Excel (Hu et al., 2018).

\section{Conclusiones}

Las unidades militares de la FAE cuentan con procesos complejos para su gestión organizacional; sin embargo, varios carecen de un nivel de automatización compatible con las tecnologías actuales, lo que posibilita la ocurrencia de errores administrativos y vulnerabilidades en la seguridad. Por esta razón es importante plantear soluciones automatizadas e integradas que contribuyan a optimizar el análisis y el intercambio de datos, así como a prevenir inobservancias a las normas legales, responsabilidades administrativas y afectaciones que comprometan los recursos humanos y materiales de la institución.

Se evidenció que las debilidades identificadas en los procesos tomados como muestra tienen su origen 
en la elevada cantidad de procedimientos manuales, los cuales generan tiempos que no aportan a la ejecución de las tareas, produciendo "cuellos de botella" y colas, de acuerdo a los resultados de la simulación. A estas afectaciones se suma la alta rotación del personal involucrado en las tareas; condiciones que son favorables para la toma de decisiones subjetivas y el cometimiento de errores o inobservancias a las normas institucionales.

La aplicación de un modelo SIG, combinando el rediseño de la gestión por procesos, la gestión de riesgos y la automatización en un entorno de base de datos integrada como el aplicado en esta investigación a través del software piloto implementado en el ala de combate ${ }^{\circ}{ }^{\circ} 21$, redujeron significativamente los tiempos de ejecución y el uso de recursos humanos y materiales en los subprocesos considerados como muestra, aun cuando se introdujo el modelo del queso suizo para la prevención de riesgos, lo que contribuyó, además, al intercambio de información con otros departamentos de la unidad militar, dando como resultado procesos administrativos y de seguridad más robustos, optimizados y modernizados.

Los subprocesos considerados como muestra para la aplicación de este modelo fueron seleccionados por su elevada complejidad y las vulnerabilidades que presentan; sin embargo esta metodología es aplicable a toda la gestión por procesos, constituyéndose como una herramienta que guía el diseño e implementación de soluciones informáticas a través de la evaluación de sus IDC para garantizar la optimización en el rendimiento de los procesos administrativos y de seguridad de las unidades militares de forma integral.

Declaración de conflicto de interés: El autor no manifiesta conflictos de interés institucionales ni personales.

\section{Referencias}

Amaechi, J., Agbasonu, V., \& Nwawudu, S. (2018). Design and Implementation of a Hospital Database Management System (HDMS) for Medical Doctors. International
Journal of Computer Theory and Engineering, 10(1), 1-6. https://doi.org/g2tp

Akuh, R., \& Atombo, C. (2019). Road transport accident analysis from a system-based accident analysis approach using Swiss cheese model. International Journal of Engineering Education, 1(2), 99-105. https://doi.org/g2t9

Askari, G., Asghri, N., Gordji, M. E., Asgari, H., Filipe, J. A., \& Azar, A. (2020). The impact of teamwork on an organization's performance: A cooperative game's approach. $M a-$ thematics, 8(10), 1-15. https://doi.org/g2tq

Benítez, G. (2013). Propuesta de diseño e implementación de un sistema de información gerencial aplicado a las operaciones aéreas y evaluación de pilotos, para el Escuadrón de Combate n. 2.313 de la Fuerza Aérea Ecuatoriana [Trabajo de grado, Escuela Politécnica del Ejército]. Repositorio institucional de la Escuela Politécnica del Ejército. http://repositorio.espe.edu.ec/xmlui/handle $/ 21000 / 1058 /$ browse?value=Gonzalo+Benítez $\% 2 C+$ Lloré\&type=author

Brooks, D. J., Coole, M., \& Haskell-Dowland, P. (2020). Intelligent building systems: Security and facility professionals' understanding of system threats, vulnerabilities and mitigation practice. Security Journal, 33(2), 244-265. https://doi.org/g2tr

Ciancio, S. (2018). The prevalence of service excellence and the use of business process improvement methodologies in Australian universities. Journal of Higher Education Policy and Management, 40(2), 121-139. https://doi.org/g2ts

Dirección de planificación y gestión estratégica de la Fuerza Aérea Ecuatoriana. (2018). Manual de procesos de la dirección de recursos humanos de la Fuerza Aérea Ecuatoriana (1.0). Fuerza Aérea Colombiana.

Evans, M., He, Y., Maglaras, L., \& Janicke, H. (2019). HEART-IS: A novel technique for evaluating human error-related information security incidents. Computers and Security, 80, 74-89. https://doi.org/g2tt

Figueroa-García, J. C., López-Santana, E. R., Villa-Ramírez, J. L., \& Ferro-Escobar, R. (2017). Automation of a Business Process Using Robotic Process Automation (RPA): A Case Study. En Applied computer sciences in engineering: 4th workshop on engineering applications, WEA 2017, 27-29, 2017 , proceedings. Communications in Computer and Information Science. https://doi.org/g2tv

Heinrich, R., Merkle, P., Henss, J., \& Paech, B. (2017). Integrating business process simulation and information system simulation for performance prediction. Software and Systems Modeling, 16(1), 257-277. https://doi.org/f9wkn3 
Hu, K., Orghian, D., \& Hidalgo, C. (2018). DIVE: A mixed-initiative system supporting integrated data exploration workflows. HILDA' 2018: Proceedings of the Workshop on Human-In-the-Loop Data Analytics, 1-7. https://doi.org/ gfw9mw

Jamil, F., Ahmad, S., Iqbal, N., \& Kim, D. H. (2020). Towards a remote monitoring of patient vital signs based on loT-based blockchain integrity management platforms in smart hospitals. Sensors, 20(8), 2195. https://doi.org/ ggvh7m

Laguna, M., \& Marklund, J. (2018). Business Process Modeling, Simulation and Design (3. ${ }^{\mathrm{a}}$ ed.). CRC Press. https://doi.org/g2tx

Larouzee, J., \& Le Coze, J. C. (2020). Good and bad reasons: The Swiss cheese model and its critics. Safety Science, 126, 104660. https://doi.org/gjm6g2

Lyon, B. K., \& Popov, G. (2020). Managing Risk Through Layers of Control. Professional Safety Journal, 65(4), 25-35. https://www.proquest.com/scholarly-journals/managing -risk-through-layers-control/docview/2398591993/se-2 ?accountid $=201395$

Mat Ghani, M. S., \& Zheng Yi, W. (2018). Implementation of Swiss Cheese for UniKL MIAT hangar. IOP Conference Series: Materials Science and Engineering, 405(1). https://doi.org/g2tz

Ministerio de Defensa Nacional. (2018a). Estatuto orgánico de gestión organizacional por procesos de la Fuerza Aérea Ecuatoriana. Gobierno de Ecuador.

Ministerio de Defensa Nacional. (2018b). Política de la defensa nacional del Ecuador "Libro Blanco". Gobierno de Ecuador. https://www.defensa.gob.ec/wp-content/ uploads/2019/01/Política-de-Defensa-Nacional-Libro-Blanco-2018-web.pdf

Nikolaidou, M., Anagnostopoulos, D., \& Tsalgatidou, A. (2001). Business processes modelling and automation in the banking sector: A case study. International Journal of Simulation: Systems, Science and Technology, 2(2), 65-76.
Noh, J. Y., Song, J. Y., Yoon, J. G., Seong, H., Cheong, H. J., \& Kim, W. J. (2020). Safe hospital preparedness in the era of COVID-19: The Swiss cheese model. International Journal of Infectious Diseases, 98, 294-296. https://doi.org/g2t2

Pérez, J. (2004). Gestión por Procesos. ESIC Editorial.

Prajogo, D., Toy, J., Bhattacharya, A., Oke, A., \& Cheng, T. C. E. (2018). The relationships between information management, process management and operational performance: Internal and external contexts. International Journal of Production Economics, 199, 95-103. https://doi.org/gdnzdd

Prolis lab2k. (2019). BPSimulator. https://www.bpsimulator. com/

Raynus, J. (2011). Improving Business Process Performance (1a ed.). Auerbach Publications. https://doi.org/bpm6cp

Reason, J. (1990). Human Error. Cambridge University Press.

Reniers, G., Landucci, G., \& Khakzad, N. (2020). What safety models and principles can be adapted and used in security science? Journal of Loss Prevention in the Process Industries, 64, 104068. https://doi.org/g2t3

Ricardo, C. M. (2009). Bases de Datos. McGraw Hill.

Sehlin, D., Truedsson, M., \& Cronemyr, P. (2019). A conceptual cooperative model designed for processes, digitalisation and innovation. International Journal of Quality and Service Sciences, 11(4), 504-522. https://doi.org/gjbjtx

Shokouhi, Y., Nassiri, P., Mohammadfam, I., \& Azam, K. (2019). Predicting the probability of occupational fall incidents: a Bayesian network model for the oil industry. International Journal of Occupational Safety and Ergonomics, 27(3), 654-663. https://doi.org/g2t4

Song, G., Khan, F., \& Yang, M. (2018). Security assessment of process facilities - Intrusion modeling. Process Safety and Environmental Protection, (117), 639-650. https://doi.org/g2t5

Van Der Aalst, W. M. P., La Rosa, M., \& Santoro, F. M. (2016). Business process management. Business \& Information Systems Engineering, 58(1), 1-6. https://doi.org/gfxx6r 